\title{
NON-ANALYTIC FUNCTIONS OF A COMPLEX VARIABLE*
}

BY E. R. HEDRICK

1. Introduction. The outlines of the theory of non-analytic functions of a complex variable, called also polygenic functions, have been stated in recent years in a number of articles. $\dagger$ Indeed, from the very first of the modern study of the theory of functions, going back at least as far as the famous inaugural dissertation of Riemann, the beginnings of the subject have been mentioned essentially, if for no other purpose than to state the conditions under which a function of a complex variable is analytic, and to delimit the field of functions to be studied.

In the present address, such preliminary details will be mentioned only briefly, with references; but enough of them must be stated to develop a notation, and to give the proper setting. More detailed attention will be given to those developments which have taken place during the last decade, and to some hitherto unpublished facts. A brief review of some of the historical background will serve both its obvious purpose, and also that of introducing the necessary preliminary details and notations.

2. Historical Background. As was stated above, every careful presentation of the classical theory of functions of a complex variable did include in a measure the elementary ideas for the general case of any function of a complex variable. A function

$$
w=f(z)=\phi(x, y)+i \psi(x, y),
$$

where $w=u+i v$ and $z=x+i y$, is said to be defined for a given region (or set of values) of $z$ if $w$ is determined whenever $z$ is assigned a value in that region (or set). The equation (1) is then equivalent, of course, to the two real simultaneous equations

$$
u=\phi(x, y), \quad v=\psi(x, y),
$$

which themselves express a transformation of the $x y$ plane onto

* An address delivered at New Orleans, December 31, 1931, as the retiring presidential address, before the American Mathematical Society.

$\dagger$ See the list of recent articles at the end of this paper. 
the $u v$ plane. In general, we shall assume that $\phi$ and $\psi$ are continuous, and that they possess continuous first partial derivatives, unless the contrary is stated.

If $z$ and $w$ have corresponding increments $\Delta z$ and $\Delta w$, the limit of their ratio, $\Delta w / \Delta z$, is the derivative $d w / d z$ whenever it has a unique value for all methods of approach of $\Delta z$ to zero, for a fixed value of $z$; in general, that limit will depend upon the slope $m$ of the curve along which $\Delta z$ approaches zero, and we shall call it the directional derivative. This limit is written in a variety of forms in standard works; we shall write

$$
\begin{aligned}
\gamma=\alpha+i \beta & =\frac{d w}{d z}=\lim _{\Delta z \rightarrow 0} \frac{\Delta w}{\Delta z}=\lim _{\substack{\Delta x \rightarrow 0 \\
\Delta y \rightarrow 0}} \frac{\Delta u+i \Delta v}{\Delta x+i \Delta y} \\
& =\frac{u_{x}+i v_{x}+m\left(u_{y}+i v_{y}\right)}{1+i m},
\end{aligned}
$$

where $u_{x}, u_{y}, v_{x}, v_{y}$ denote the partial derivatives in the usual manner, and where $m=d y / d x$ is the slope of the curve of approach. In order that (3) give a unique result independent of $m$, the usual necessary and sufficient conditions are the well known Cauchy-Riemann equations

$$
u_{x}=v_{y}, \quad u_{y}=-v_{x} .
$$

The equation (3) may be written in other forms. Riemann, in his inaugural dissertation, wrote it essentially in the following form. Let $\theta$ denote the angle between the $x$ axis and the curve of approach, so that $\tan \theta=m$; then, by means of the usual relation $e^{i \theta}=\cos \theta+i \sin \theta$, it is easy to reduce (3) to the form

$$
\gamma=\frac{d w}{d z}=\lim _{\Delta z \rightarrow 0} \frac{\Delta w}{\Delta z}=\mathcal{D}[f(z)]+\mathcal{P}[f(z)] e^{-2 i \theta},
$$

where

$$
\begin{aligned}
& \mathcal{D}[f(z)]=\frac{1}{2}\left[u_{x}+v_{y}+i\left(v_{x}-u_{y}\right)\right], \\
& \mathcal{P}[f(z)]=\frac{1}{2}\left[u_{x}-v_{y}+i\left(v_{x}+u_{y}\right)\right] .
\end{aligned}
$$

These expressions will occur frequently in what follows. The expression $\mathcal{D}(w)$, which is the part of (5) that is independent of $\theta$, is precisely the value of the derivative $d w / d z$ whenever the derivative has a unique value at the point $z$, that is, whenever the 
result in (5) is independent of $\theta$. The expression for $P(w)$ vanishes when and only when the equations (4) hold. It is pointed out that some of the results recently announced can be seen as immediate consequences of (5).

The preceding classical statements evidently do not presume in advance that $w$ is an analytic function of $z$, nor that the equations (4) hold. This is true of many other results of the classical theory. In particular, the well known Morera Theorem,* the Weierstrass Mean-Value Theorem, $\dagger$ and many others, do not presume the analytic character of the functions in their hypotheses. In such theorems as that of Morera, in fact, the very point of the theorem would be lost if there were such an original hypothesis.

Specific attempts to discuss as such functions that are not analytic have been made from time to time. One such that I would mention is a paper by Picard, $\ddagger$ in which the attention is directed toward generalizing the Cauchy-Riemann equations (4).

The expression $P(w)$ defined in (6) is equal, except for a constant factor (see §9), to a concept considered by D. Pompeiu, and later by Hayashi. $\S$ Pompeiu arrived at this concept, which he called the areal derivative, by considering the limit

$$
\lim _{C=0} \frac{\int_{C} f(z) d z}{\frac{1}{2} \int_{C} x d y-y d x}=\lim _{C=0} \frac{\int_{C} f(z) d z}{A}
$$

where $C$ denotes a contour surrounding the point $P, A$ the area of the region bounded by $C$, and where the limit is taken as $C$ shrinks toward $P$ in such a way that $C$ eventually lies in an arbitrarily small circle about $P$. Some of the results of these papers are stated below $(\$ 9)$.

* Morera, Rendiconti, Istituto Lombardo, (2), vol. 19 (1886); and texts on the classical theory.

† See, for example, Osgood, Lehrbuch der Funktionentheorie, 1923, vol. 1, p. 212; Goursat, Mathematical Analysis (English ed.), vol. 2, Part 1, p. 65.

$\ddagger$ Journal de Mathématiques, (4), vol. 8 (1892), p. 217.

$\S$ D. Pompeiu, Rendiconti di Palermo, vol. 33 (1912), pp. 108-113, and vol. 35 (1913), p. 277. 
The other papers cited at the end of this address, and still others, have appeared during the past decade. They form together a body of knowledge concerning this field of which the remainder of this address gives a summary.

3. Principal Directions. Characteristic Lines. If we introduce the familiar notation

$$
E=u_{x}^{2}+v_{x}^{2}, \quad G=u_{y}^{2}+v_{y}^{2}, \quad F=u_{x} u_{y}+v_{x} v_{y},
$$

it follows readily* that

(9) $r=\left|\frac{d w}{d z}\right|^{2}=\left|\lim _{\Delta z \rightarrow 0} \frac{\Delta w}{\Delta z}\right|^{2}=\frac{d u^{2}+d v^{2}}{d x^{2}+d y^{2}}=\frac{E+2 m F+G m^{2}}{1+m^{2}}$,

where $m$ denotes, as before, the slope $d y / d x$ of the curve of approach. The maximum and minimum values of $r$ are found to exist for values of $m$ given by the equation

$$
F+(G-E) m-F m^{2}=0,
$$

and these values of $r$ are themselves solutions of the quadratic equation

$$
\rho^{2}-(E+G) \rho+J^{2}=0,
$$

where

$$
J=\left(E G-F^{2}\right)^{1 / 2}=u_{x} v_{y}-u_{y} v_{x} .
$$

Since the roots of (10) are negative reciprocals, it follows that the directions which give a maximum and a minimum stretching ratio $R=r^{1 / 2}$, are at right angles. These directions we called the principal directions. They were discovered first by Tissot $\dagger$ in his study of the mapping problem. The two families of curves that are tangent to these principal directions at every point we called the characteristic curves for the given function.

It is shown $\ddagger$ that the only orthogonal system of curves in the z plane which corresponds by the transformation $w=f(z)$ to an orthogonal system in the w plane, is the system of characteristic lines.

If we lay off, at a fixed point $z_{0}$, in the direction $m$, the recipro-

* See Hedrick, Ingold, and Westfall, loc. cit.

$\dagger$ Tissot, Sur les cartes géographiques, Comptes Rendus, 1849.

$\ddagger$ Hedrick, Ingold, and Westfall, loc. cit. See also the geometric proof given by Darboux, Lȩ̧ons sur la Théorie des Surfaces, vol. 3, p. 49. 
cal stretching factor $P=1 / R$, the curve obtained* is the ellipse

$$
E \bar{x}^{2}+2 F \bar{x} \bar{y}+G \bar{y}^{2}=1,
$$

where $\bar{x}, \tilde{y}$ are the coordinates measured from the point $z_{0}$ as origin. This ellipse may be called the Tissot indicatrix. Similarly, if (2) can be solved for $x$ and $y$ in terms of $u$ and $v$, the factor $R$ can be laid off about the point $w_{0}$ in the $w$ plane to obtain the corresponding ellipse

$$
\varepsilon_{\bar{u}^{2}}+2 \mathcal{f} \bar{u} \bar{v}+G^{\bar{v}^{2}}=1
$$

in the $u v$ plane, where $\mathcal{E}, \mathcal{F}, G$ are the fundamental quantities similar to $E, F, G$ when $u$ and $v$ are the independent variables.

It is clear that these concepts all degenerate, the ellipses becoming circles and the principal directions becoming indeterminate, if

$$
E=G, \quad F=0 .
$$

These equations are satisfied, of course, if the Cauchy-Riemann equations (4) are satisfied, that is, if $f(z)$ is an analytic function of $z$, or also if $f(z)$ is an analytic function of $\bar{z}=x-i y$, in which case the signs in (4) are changed, but (14) still hold.

4. Pointwise Analytic Functions. It is evident that the derivative $d w / d z$ may exist at isolated points in the sense that the limit (3) may be independent of $m$ at isolated points. These points are the solutions of (4) regarded as simultaneous equations for $x$ and $y$. We say that $f(z)$ is analytic at a point $z_{0}$ if (4) hold at that point.

Since we have assumed that $u$ and $v$, and their first derivatives, are continuous, it follows that the points at which $f(z)$ is analytic form a closed set, except for points at which $f(z)$ is not defined or does not satisfy these hypotheses. At all points at which $f(z)$ is analytic, the ellipses defined in $\$ 3$ become circles and the principal directions become indeterminate.

5. The Beltrami Equations. Classification of Functions. The Cauchy-Riemann equations (4) have been generalized by Beltrami $\dagger$ to the case of any surface. These equations are

* Hedrick, National Academy of Sciences, loc. cit.

$\dagger$ Beltrami, Delle variabili complesse sopra una superficie qualunque, Annali di Matematica, (2), vol. 1; see also Picard, Traité d'Analyse, vol. 2, p. 8. 


$$
\begin{aligned}
& J v_{x}=F u_{x}-E u_{y}, \\
& J v_{y}=G u_{x}-F u_{y} ;
\end{aligned}
$$

and $u$ and $v$ satisfy the generalized Laplace equation

$$
\frac{\partial}{\partial x}\left(\frac{F U_{y}-G U_{x}}{J}\right)+\frac{\partial}{\partial y}\left(\frac{F U_{x}-E U_{y}}{J}\right)=0 .
$$

These equations, which evidently hold for the plane also, are here written with the symbol $J=\left(E G-F^{2}\right)^{1 / 2}$, instead of the symbol $H$ employed in the theory of surfaces.

As in the case of a surface, so here also, those functions for which the ratios $E: F: G$ are the same form a class. Every function $f(z)$ belongs to some class; no function belongs to two different classes; there exist functions belonging to every class; and if two functions belong to the same class, the one is an analytic function of the other.

It is easy to prove* that two functions $f=u+i v$ and $F=U+i V$ do belong to the same class, so that one is an analytic function of the other, if and only if the jacobian of $F$ and $f$ with respect to $x$ and $y$ vanishes :

$$
\left|\begin{array}{cc}
\frac{\partial(U+i V)}{\partial x} & \frac{\partial(U+i V)}{\partial y} \\
\frac{\partial(u+i v)}{\partial x} & \frac{\partial(u+i v)}{\partial y}
\end{array}\right|=0 .
$$

For functions of the same class, a function theory entirely analogous to that of ordinary analytic functions exists.

6. The Kasner Circle. Let us now return to the formula for the directional derivative, which we write in the form

$$
\gamma=\alpha+i \beta=\left.\frac{d w}{d z}\right|_{\theta}=\mathcal{D}+\mathscr{P} e^{-2 i \theta},
$$

where $\mathcal{D}$ and $\mathcal{P}$ are functions of $z$ alone, independent of $\theta$, and are defined by the equations (6). It is immediately evident from (18) that the values of the directional derivative $\gamma$, for a fixed value of $z$, depend only on $\theta$, and that these values of $\gamma$ all lie on $a$

* See Nicolesco, Thesis, loc. cit. 
circle in the $\gamma$ plane, whose center is the point $\mathcal{D}(w)$ and whose radius is $|P(w)|$. In fact, (18) can be written in the form

$$
\gamma-\mathcal{D}=\mathcal{P} e^{-2 i \theta}
$$

whence

$$
|\gamma-\mathcal{D}|=|\mathcal{P}|
$$

which is the equation of the circle described above. Here $\gamma-\mathcal{D}$ is a vector counted from the point $\mathcal{D}$ as origin, which is a fixed point if $z$ is fixed. If we insert the detailed values given by (6), and the value $\alpha+i \beta$ for $\gamma$, the equation (20) becomes

$$
\begin{gathered}
{\left[\alpha-\left(\frac{u_{x}+v_{y}}{2}\right)\right]^{2}+\left[\beta-\left(\frac{v_{x}-u_{y}}{2}\right)\right]^{2}} \\
=\left(\frac{u_{x}-v_{y}}{2}\right)^{2}+\left(\frac{v_{x}+u_{y}}{2}\right)^{2} .
\end{gathered}
$$

In this form, the equation was first published by Kasner. ${ }^{*}$ I have proposed that the circle be called the Kasner circle. His original derivation of the equation (21) is not so immediate, however, as is the process given above; all that is necessary is to transpose the term $\mathcal{D}$ in the Riemann equation (5) and then eliminate $\theta$ by taking the absolute values on both sides.

It is obvious also from (19) that, as $\theta$ varies in the $z$ plane, the point $\gamma$ moves around the circle at twice the rate at which $\theta$ changes, and in the opposite sense.

On account of the resemblance just described to an ordinary clock-motion, Kasner has proposed that the distribution of the values of the directional derivative on this circle be called a clock. As $z$ varies, there will be one such clock for every value of $z$ in the $z$ plane; Kasner has called the whole family a congruence of clocks. He has pointed out also $\dagger$ that the mean value of $\gamma$ on the circle is

$$
\begin{aligned}
\frac{1}{\pi} \int_{0}^{\pi} \gamma d \theta & =\frac{1}{\pi} \int_{0}^{\pi}(\alpha+i \beta) d \theta \\
& =\frac{1}{\pi} \int_{0}^{\pi}\left(\mathcal{D}+\mathcal{P} e^{-2 i \theta}\right) d \theta=\mathcal{D} ;
\end{aligned}
$$

* Kasner, Science, vol. 66 (1927), pp. 581-582.

$\dagger$ Kasner, National Academy of Sciences, loc. cit., p. 81. 
and he calls $\mathcal{D}(w)$ the mean derivative of $w$. The reason for this result is simply that $\mathcal{D}$ and $P$ are constant during this integration, since only $\theta$ varies; and since

$$
\int_{0}^{\pi} P e^{-2 i \theta} d \theta=P \int_{0}^{\pi} e^{-2 i \theta} d \theta=0,
$$

the term involving $P$ in (22) falls out, and (22) becomes an identity.

Other developments regarding the congruence of clocks as a picture of the directional derivative are given by Kasner (loc. cit.) and by Kasner and Hofmann.*

7. Riemann Surfaces. The Increment Ratio. It is well known that any function $w=f(z)$, that is, any transformation of the form (2), can be represented by a Riemann surface. The equations (2) can be solved for $x$ and $y$ under the hypotheses made above, near any point $\left(x_{0}, y_{0}\right)$ at which the jacobian $J=u_{x} v_{y}$ $-u_{y} v_{x}$ does not vanish. If there are several solutions near $\left(u_{0}, v_{0}\right)$, they can be represented geometrically by points of a Riemann surface of several sheets over the $u v$ plane, as in the usual theory. These sheets must be connected, if at all, along the branch curves $K$ in the $u v$ plane, which are curves that correspond to the critical curve

$$
J(x, y)=0
$$

in the $x y$ plane. The ordinary branch points of the classical theory of functions are degenerate cases of these branch curves, since the jacobian reduces to a sum of squares if the CauchyRiemann equations (4) hold. Any curve in the xy plane that passes through a point of the curve (24) corresponds to a curve in the uv plane that is tangent to the corresponding branch curve $K$, unless the corresponding point $(u, v)$ is a singular point of $K . \dagger$

On account of these properties, I have proposed to call the branch curves $K$ the edge of regression on the Riemann surface, since they play a role very similar to that of the edge of regression of a developable surface. Indeed, if any family of curves

$$
x=p(t, \alpha), \quad y=q(t, \alpha),
$$

* This Bulletin, vol. 34 (1928), pp. 495-503.

$\dagger$ Hedrick, Bulletin of the Calcutta Society, loc. cit., p. 114. 
crosses the critical curve $C$ at a variable point that is a function of $\alpha$, the edge of regression $K$ is the envelope of the corresponding family in the uv plane.

I have stated some examples in my Calcutta paper (loc. cit.). The usual derivative ratio

$$
\zeta=\xi+i \eta=\frac{\Delta w}{\Delta z}=\frac{\Delta u+i \Delta v}{\Delta x+i \Delta y}
$$

is a single-valued function of $z=z_{0}+\Delta z$ for a fixed point $z_{0}$, except at the point $z=z_{0}$, at which it is not defined. I have shown in my Calcutta paper that the jacobian of $\zeta$

$$
J=\xi_{x} \eta_{y}-\xi_{y} \eta_{x}
$$

vanishes at precisely the points of the Kasner circle. Hence the Kasner circle is precisely the edge of regression for the function $\zeta$, which is in general represented on a two-leaved Riemann surface whose leaves join along the Kasner circle. As the point z revolves once around the point $z_{0}$, the point $\zeta$ revolves twice about the Kasner circle.

This fact makes it very evident why the directional derivative $\gamma$ revolves on the Kasner circle twice as fast as the angle $\theta$ revolves in the $z$ plane; the directional derivative is indeed precisely the edge of regression, geometrically, and is the missing definition of what corresponds in (25) to the value $z=z_{0}$.

8. Other Geometric Properties. Several particular cases, and particular values of the directional derivative $\gamma$ on the circle, are given by Kasner. ${ }^{*}$ He uses the fact that the point $A\left(v_{y}, v_{x}\right)$ lies on the circle. Other important special points on this circle are $E\left(u_{x}, v_{x}\right), D\left(v_{y},-u_{y}\right), B\left(u_{x},-u_{y}\right)$. In my Calcutta paper, I have used these four points $A, E, D, B$, which evidently lie at the vertices of a rectangle inscribed in the circle. From the origin $O$ in the plane of $\gamma$, the distances $O A$ and $O E$ are

$$
O A^{2}=v_{x}^{2}+v_{y}^{2}=G, O E^{2}=u_{x}^{2}+u_{y}^{2}=E,
$$

which represent the two fundamental quantities $E$ and $G$. It is also easy to represent the fundamental quantity $F$ as the difference between the areas of two rectangles in the same figure. I

* Kasner, Proceedings of the National Academy, vol. 14 (1928), pp. 75-82. 
have pointed out also that the diameter through $O$ has its extremities at points $\gamma_{1}$ and $\gamma_{2}$ of the Kasner circle, so that $\left|\gamma_{1}\right|$ and $\left|\gamma_{2}\right|$ are precisely the maximum and the minimum values of $|\gamma|$, that is, the maximum and minimum values of the stretching ratio

$$
R=r^{1 / 2}=|\gamma|=\left|\frac{d w}{d z}\right|,
$$

discussed in $\S 3$. Finally, I have shown that the jacobian of $f(z)$ is represented by the length of the tangent from $O$ to the Kasner circle. The jacobian of $f(z)$ can vanish, therefore, only for values of $z$ for which the Kasner circle passes through the origin $O$ in the $\gamma$ plane. It follows that the jacobian vanishes at a point $z$ if and only if at least one determination of the directional derivative $\gamma$ vanishes at that point. This can be verified also immediately by substituting $\alpha=0, \beta=0$ in the equation (21).

9. The Areal Derivative. By Green's Theorem, we may write

$$
\int_{C} f(z) d z=\int_{C}(u+i v)(d x+i d y)=2 i \iint_{R} P[f(z)] d x d y,
$$

where $C$ is a closed curve, and where the double integral is taken over the region $R$ bounded by $C$. If $\sigma$ denotes the area of $R$, we have, by the law of the mean,

$$
\int_{C} f(z) d z=2 i \Phi[f(\zeta)] \iint_{R} d x d y=2 i \Phi[f(\zeta)] \cdot \sigma,
$$

where $\zeta$ is some value of $z$ in $R$. If $C$ shrinks toward a single point $z, \zeta$ also approaches $z$, and we may write

$$
2 i \Phi[f(z)]=\lim \frac{1}{\sigma} \int_{C} f(z) d z .
$$

This expression is defined by Pompeiu* to be the areal derivative.

If we introduce the conjugate imaginary quantity $\bar{z}=x-i y$, we may find, similarly,

\footnotetext{
* Pompeiu, Rendiconti di Palermo, vol. 33 (1912), p. 112. I have used the symbol $\mathcal{P}$ because it is his initial.
} 


$$
2 i \mathcal{D}[f(z)]=\lim \frac{1}{\sigma} \int_{C} f(z) d \bar{z} .
$$

Hence the formula (5) may be written in the form

$$
\text { (30) } \gamma=\frac{d w}{d z}=-\frac{i}{2} \lim \frac{1}{\sigma}\left[\int_{C} f(z) d \bar{z}+e^{-2 i \theta} \int_{C} f(z) d z\right] \text {. }
$$

It may be remarked that the forms (28), (29), (30), and hence also the forms (26) and (27), can be interpreted even when it is not supposed by hypothesis that the first derivatives of $u$ and $v$ exist. Some results exist in this field, but they are not important. I propose, however, to pursue this and to investigate results obtainable without the hypothesis in question. It is to be remarked that the results of $\$ 10$, and the well known theorem of Goursat mentioned there, may be construed to be theorems of this category.

Pompeiu, Hayashi, and Nicolesco* have considered at length the values of $\int f(z) d z$ on a circle of radius $\rho$ about the point $z_{0}$; and have derived formulas for the expansion of this integral in terms of successive powers of $\rho^{2}$. From these expansions, several theorems are deducible. Thus, if $\left(1 /\left(\pi \rho^{2}\right)\right) \int f(z) d z$, taken over concentric circles of radius $\rho$, is independent of $\rho$, the function is called by Nicolesco non-analytic of the first degree. It is shown by Hayashi that for such a function $\nabla^{2} u+i \nabla^{2} v$ is itself an analytic function, where $\nabla^{2}$ denotes the usual Laplace operator. In $\$ 10$, we shall see that if the same expression approaches zero with $\rho$, for a set of values of $z$ that is everywhere dense, then $f(z)$ is an analytic function.

The introduction of the conjugate imaginary $\bar{z}$ may be employed to simplify the notations as follows. Using the simple identities

we may write

$$
x=\frac{z+\bar{z}}{2}, \quad y=\frac{z-\bar{z}}{2 i}
$$

$$
\begin{aligned}
w=f(z) & =\phi\left(\frac{z+\bar{z}}{2}, \frac{z-\bar{z}}{2 i}\right)+i \psi\left(\frac{z+\bar{z}}{2}, \frac{z-\bar{z}}{2 i}\right) \\
& =F(z, \bar{z}),
\end{aligned}
$$

\footnotetext{
* Pompeiu, loc. cit.; Hayashi, loc. cit.; Nicolesco, Thesis, loc. cit.
} 
whence, if we agree to differentiate partially* with regard to $z$ and $\bar{z}$, we may write, by an easy identification with (6),

$$
\frac{\partial w}{\partial z}=\mathcal{D}(w), \quad \frac{\partial w}{\partial \bar{z}}=P(w),
$$

and $\dagger$

$$
\gamma=\frac{d w}{d z}=\frac{\partial w}{\partial z}+e^{-2 i \theta} \frac{\partial w}{\partial \bar{z}} .
$$

In order to refer by some name to $P(w)$, I shall call it the conjugate derivative, for the reason suggested by (32), and because the name is otherwise appropriate; whenever it is convenient to do so, however, I shall use the combination $2 i P(w)$, which is the Pompeiu areal derivative. Similarly, I shall call the integral $\int f(z) d \bar{z}$ the conjugate integral; for the most part, however, I shall use the mean derivative $\mathcal{D}(w)$, which, by (29), differs from this conjugate integral only by the factor $2 i$.

Pompeiu considered the integral

$$
\int_{C} \frac{f(z)}{z-\zeta} d z
$$

where $\zeta$ is any point in $R$; and he obtained the formula

$$
f(z)=\frac{1}{2 \pi i} \int_{C} \frac{f(s)}{s-z} d s+\frac{1}{\pi} \iint_{R} \frac{P[f(s)]}{z-s} d x d y,
$$

which is the generalization of the well known Cauchy Integral Theorem. Essentially the same formula is used by Borel in his Leçons sur les Fonctions Monogènes, and by Calugaréano in his thesis, where a complete proof is given. This formula shows that a function $f(z)$ is completely determined in $R$ if its values are known on $C$, and if the areal derivative is known in $R$. The classical theorem is a special case of this one, since in the classical case the areal derivative is known to be identically zero.

The first term of (34), as is well known in the classical theory,

* Calugaréano, Thesis, p. 5, credits this suggestion to Al. Proca, who has himself not published it; such forms are used also by Nicolesco in his thesis.

$\dagger$ We may even write, with Calugaréano, $d w / d z=\partial w / \partial z+(\partial w / \partial \bar{z})(d \bar{z} / d z)$, since it is true that $d \bar{z} / d z=e^{-2 i \theta}$. 
represents a function analytic in $R$. It follows that the knowledge of the areal derivative in $R$ determines a function $f(z)$ to within an analytic function. This follows also from the fact that if two functions $f_{1}$ and $f_{2}$ have the same areal derivative, the areal derivative of their difference is zero; that is, their difference is an analytic function. Calugaréano shows in his thesis (pp. 15-20) that the areal derivative can be assigned arbitrarily; that is, if $P(w)$ be any function of $z, f(z)$ given by the second term of (34) has $P(w)$ as its areal derivative.

Since the term $e^{-2 i \theta} \mathcal{P}(w)$ in (5) essentially determines what Kasner has called a clock, the same theorems hold, by mere rewording, in terms of the concept of clock.

10. The Morera Theorem. The original Morera Theorem is contained in all standard works on the classical theory of functions. Refinements of the hypotheses have been made by several authors. H. Looman* has proved the following theorem.

If $z$ is a point of a region $R$, and $\gamma$ is a square containing $z$, consider the function

$$
\sigma(z)=\limsup _{\gamma \rightarrow 0} \frac{\int_{\gamma} f(z) d z}{\gamma},
$$

where $\gamma$ denotes also the area of the square; if then $\sigma(z)$ is everywhere finite and equal to zero almost everywhere, the function $f(z)$ is analytic throughout $R$.

A further extension of this theorem was given by J. Wolff. $\dagger$ These theorems may be regarded as extensions of the well known proof by Goursat $\ddagger$ of the Cauchy Integral Theorem.

If we assume that the limit contained in Looman's theorem exists, we have in it again essentially the areal derivative of Pompeiu, so that $\sigma(z)$ is in that case equal to the areal derivative. With the assumptions regarding continuity made in the present paper, we may state the following theorem.

With the notation and assumptions of $\$ 2$, if

* Nieuw Archief voor Wiskunde, vol. 14 (1924), pp. 234-239.

$\dagger$ Report of the Toronto Congress, pp. 457-459.

$\ddagger$ Transactions of this Society, vol. 1 (1900), p. 14. 


$$
P(w)=\lim \frac{i}{2 \gamma} \int_{\gamma} f(z) d z
$$

is zero at any set of points everywhere dense in $R$, then $f(z)$ is analytic throughout $R$.

As a corollary, we may state the following generalization of the Morera Theorem.

With the notation and assumptions of $\$ 2$, if $I_{i}=\int_{C_{i}} f(z) d z a p$ proaches zero with $i$ on a set of curves $C_{i}$ that approach $z$, for each of a set of points that is everywhere dense in $R$, then $f(z)$ is analytic throughout $R$.

11. The Liouville Theorem. I have pointed out* that the familiar theorem that the absolute value of an analytic function cannot have a maximum in any region in which it is regular, can be extended to the general case of $\$ 2$. For if we consider simply the real transformation (2), and if $\left(u_{0}, v_{0}\right)$ corresponds to $x=0$, $y=0$, the existence of a maximum of $|f(z)|=u^{2}+v^{2}$ at any point $\left(u_{1}, v_{1}\right)$ would imply that the equations (2) could not be solved to give a unique solution

$$
x=\Phi(u, v), \quad y=\Psi(u, v),
$$

in a region about $\left(u_{1}, v_{1}\right)$. By the usual theorems on the existence of implicit functions, this would imply that the jacobian of (2) vanishes at $\left(u_{1}, v_{1}\right)$. If we exclude the vanishing of the jacobian (which corresponds to a requirement that the derivative shall not vanish), the maximum cannot exist. We may therefore state the following theorem.

If the jacobian of (2) does not vanish in a region $R,|f(z)|$ cannot be at a maximum at any point of $R$.

Since, as I have pointed out in $\$ 8$, the jacobian cannot vanish unless at least one value of the directional derivative at the same pcint is zero, and conversely, we may re-state this theorem as follows.

If the directional derivative $\gamma$ does not vanish in a region $R$, $|f(z)|$ cannot be at a maximum at any point of $R$.

We may use this result to generalize the classical theorem of Liouville, that every integral analytic function whose absolute

* This Bulletin, vol. 36 , p. 59 , and vol. 36, p. 801. 
value is always less than a fixed number $M$ is a constant. For, suppose that any function $f(z)$ is defined, and that the assumptions of $\$ 2$ hold, for every point $z$ in the $z$ plane, that $|f(z)|<M$ for all values of $z$, and suppose that $\lim _{z \rightarrow \infty} f(z)=0$. Then $|f(z)|$ would have a maximum at the same point in the plane, which contradicts the preceding theorem, except in the trivial case $f(z) \equiv 0$. Next, suppose that $\lim _{z \rightarrow \infty} f(z)=C$; then it is sufficient to consider the new function $\phi(z)=f(z)-C$, for which $\lim _{z \rightarrow \infty}$ $\phi(z)=0$. Hence we may state the following theorem.

If $f(z)$ satisfies the assumptions of $\$ 2$ at every point of the $z$ plane, if the directional derivative is never zero, if $|f(z)|<M$, and if $\lim _{z \rightarrow \infty} f(z)$ exists, then $f(z)$ is a constant.

As in the classical theory, it is easy to define the point at infinity, by means of the simple transformation $z=1 / z^{\prime}$. In the case just discussed, that is, when $\lim _{z \rightarrow \infty} f(z)=C$, the function $f\left(1 / z^{\prime}\right)$ is regular near the new origin $z^{\prime}=0$ if we define it to be equal to $C$ at that new origin. We may also use the classical language, and say that $f(z)$ has the value $C$ at the point at infinity, and that it is defined at every point on the "sphere," in the usual sense. Since the theorems announced above are also valid near the origin in the $z^{\prime}$ plane, we may state the following theorem, which is a generalization of one form of the Liouville theorem.

If $f(z)$ satisfies the assumptions of $\$ 2$ at every point of the $z$ sphere, and if the directional derivative is never zero, then $f(z)$ is a constant.

It is possible to replace the condition that $\lim _{z \rightarrow \infty} f(z)$ exist by lighter requirements. If $|f(z)|$ assumes the value $M$ at any point, it is easy to see that the theorem regarding the non-existence of a maximum would have to be violated at that point, if we maintain the assumption that the directional derivative never vanishes. It is indeed sufficient to assume that there exists some closed curve inside of which $f(z)$ takes on values greater than any values assumed on the curve itself.

Somewhat similar theorems may be found also by using the formula (34) in a manner analogous to the use made of the analogous theorem in the classical theory, for special hypotheses regarding the second term, that is, regarding the areal deriva- 
tive. This is precisely what is done in the classical theory, for it is assumed there that the areal derivative is identically zero. If we assume that $|f(z)|$ has a maximum $M$ at $z_{0}$, we may take $C$ to be a circle of radius $\rho$ about $z_{0}$, and we can then show from (34) that we must have

$$
\begin{aligned}
\left|f\left(z_{0}\right)\right| & \leqq \frac{1}{2 \pi} \int_{0}^{2 \pi}|f(s)| d \theta+\left|\frac{P[f(\zeta)]}{\pi}\right| \iint d \rho d \theta \\
& \leqq\{\text { Max. of }|f(z)| \text { on } C\}+2 \rho \Phi[f(\zeta)],
\end{aligned}
$$

where $\zeta$ is a value of $z$ within $C$.

12. The Derivative on a Field. Stieltjes Integrals. Nicolesco in his thesis (pp. 21-25) has proposed the idea of the derivative of $f(z)$ on a given field. This field may be given by means of a family of curves

$$
x=\phi(t, \alpha), \quad y=\psi(t, \alpha),
$$

of which one and only one passes through a given point of $R$, or by a given real function $m(x, y)$ which determines a direction $m$ at every point of $R$; that is, essentially by means of a differential equation. We shall assume the essential equivalence of these two methods of determining the field. Nicolesco has proved the following theorem.

If $m(x, y)$ is given, and if any function $\gamma(z)=\gamma=\alpha+i \beta$ is given, there exist an infinite number of functions $f(z)=u+i v$ whose derivatives on the field $m$ are equal to $\gamma(z)$; and these are the solutions of the equations

$$
\begin{aligned}
& \frac{\partial u}{\partial x}+m \frac{\partial u}{\partial y}-\alpha+m \beta=0, \\
& \frac{\partial v}{\partial x}+m \frac{\partial v}{\partial y}-m \alpha-\beta=0,
\end{aligned}
$$

in which the variables $u$ and $v$ are separated.

The functions $f(z)$ may be called the primitive functions of the function $\gamma(z)$.

I have pointed out elsewhere* that integration and differentiation can be carried out on such a field, and that they are in-

* This Bulletin, vol. 34 (1928), p. 150; and Bulletin of the Calcutta Mathematical Society, vol. 20 (1928), pp. 109-124. 
verse operations in the usual sense, if the directions taken in each case lie in the field. In fast, if (37) define the directions in question, $d x / d t=\cos \theta$ and $d y / d t=\sin \theta$ are determined at every point, so that $\theta=\theta(x, y)$ is a function of $x$ and $y$. Then, given $w=f(z)=u(x, y)+i v(x, y)$,

(39) $\gamma=\left.\frac{d w}{d z}\right|_{\theta}=\alpha+i \beta=\frac{\left(u_{x}+i v_{x}\right) \cos \theta+\left(u_{y}+i v_{y}\right) \sin \theta}{\cos \theta+i \sin \theta}$.

The integral, which I have called a Stieltjes integral, of this function $\gamma$ on the curves of the family (37) is

$$
\begin{aligned}
\int_{z_{0}}^{z} \gamma d z & =\int_{x_{0}, y_{0}}^{x, y}(\alpha+i \beta)(d x+i d y) \\
& =\int(\alpha+i \beta)\{d \phi(t, \alpha)+i d \psi(t, \alpha)\} \\
& =\int(\alpha+i \beta)(\cos \theta+i \sin \theta) d \theta \\
& =\int\left\{\left(u_{x}+i v_{x}\right) \cos \theta+\left(u_{y}+i v_{y}\right) \sin \theta\right\} d \theta \\
& =\int\left(u_{x}+i v_{x}\right) d x+\left(u_{y}+i v_{y}\right) d y=[u+i v]_{x_{0}, y_{0}}^{x, y}
\end{aligned}
$$

where the limits to be taken in the several integrals are obvious.

Conversely, the derivative on the field of the integral on the field is readily seen to be equal to the original function. For a given field, therefore, differentiation and integration may be regarded as inverse operations, the result in each case being a function of $z$ alone.

13. Second and Higher Derivatives. The definitions of second and higher derivatives that have been given by different authors are not in agreement. In taking a second derivative, if the slope of the curve along which the increment $\Delta z$ approaches zero be denoted by $m^{\prime}$, while $m$ still denotes the similar slope for the first derivative, the following plans may be (and have been) used:
(A) $m=m^{\prime}=$ const., independent of $z$.
(B) $m=m^{\prime}=\mu(x, y)$, a field (see $\S 12$ ).
(C) $m^{\prime}$, and the curve of approach, independent of $m$. 
In his thesis, Nicolesco considers the various possibilities. First of all, he proves that in any case the order of differentiation is immaterial; that is, that $m$ and $m^{\prime}$ may be interchanged without affecting the second derivative. He extends this result to the $n$th derivative. His position usually, however, is that of the assumption (A) above.

Calugaréano, who makes much more extensive use of second and higher derivatives, makes the assumption (A) throughout. He discusses the difference between his own attitude and that of Kasner in a recent paper.* We shall return to this discussion. Calugaréano proceeds with essentially the same notation as that used in equations (31), (32), (33) of $\$ 9$ of the present paper. Assuming $m=m^{\prime}$, we easily derive the formula

$$
\frac{d^{n} w}{d z^{n}}=\left(\frac{\partial}{\partial z}+e^{-2 i \theta} \frac{\partial}{\partial \bar{z}}\right)^{(n)} w,
$$

where the symbolic exponent $(n)$ is to be interpreted in the usual symbolic sense. The simplicity of this formula makes unnecessary any extended discussion, if this interpretation is to be adopted.

Kasner $\dagger$ and his students $\ddagger$ have adopted the assumption (C). He uses as the path of approach any curve whatever, independent of the original choice of $m$, and he obtains the formula

$$
\frac{d^{2} w}{d z^{2}}=\frac{w_{x x}+2 w_{x y} y^{\prime}+w_{y y} y^{\prime 2}}{\left(1+i y^{\prime}\right)^{2}}+\frac{w_{y}-i w_{x}}{\left(1+i y^{\prime}\right)^{3}} y^{\prime \prime} .
$$

This formula depends essentially upon the second derivative $y^{\prime \prime}$ of the curve of approach, that is, upon the curvature of that curve. He shows that $d^{2} w / d z^{2}$ is independent of $y^{\prime \prime}$, under his assumptions, when and only when $w$ is an analytic function of $z$. He also proves that the $\infty^{1}$ set of values of $d^{2} w / d z^{2}$, for a constant curvature $\kappa$, describe an irrational curve of the eighth order, which reduces to a limaçon when $\kappa=0$. A detailed report upon these facts seems inappropriate here, though the geometry developed is of great interest. Further developments of this

* Transactions of this Society, vol. 32 (1930), pp. 110-113.

$\dagger$ Transactions of this Society, vol. 30 (1928), pp. 803-818.

$\ddagger$ Kasner and Hofmann, this Bulletin, vol. 34 (1928), p. 495; J. E. Donahue, Columbia University Thesis, Hamburg, 1930. 
geometry have been given by J. E. Donahue in his thesis (loc. cit.). He includes a review of Kasner's work (only), and gives elaborate details with many special cases, and with very interesting geometric developments. These seem, however, to be beyond the scope of this paper.

14. Solutions of Differential Equations. Calugaréano, using the definitions stated here in $\S 13$, has proceeded to discuss the non-analytic solutions of differential equations and of functional equations. It will not be possible here, in the space at my command, to do more than to indicate the nature of his results, and to state a few of the simplest theorems. It may be said that these developments would not be possible under the definitions assumed by Kasner, and that it would seem that differential equations of the sort discussed below would not possess nonanalytic solutions if the Kasner definition were to be used.

Returning to the definition used by Calugaréano, we may say that a differential equation

$$
\Phi\left(z, w, w^{\prime}, \cdots, w^{(n)}\right)=0
$$

will be satisfied, in general, by a function $w=f(z)$ if, when we substitute for the $n$th derivative $w^{(n)}$ the value given in (41), the equation (43) is satisfied for all values of $\theta$. An attempt to express the fact that the result of the substitution is independent of $\theta$ gives in general a number of conditions which must be satisfied if there are to be any non-analytic solutions. That nonanalytic solutions do actually exist in some cases is easy to verify. Thus the linear equation

$$
A(z) w^{\prime \prime}+B(z) w^{\prime}+C(z) w+D(z)=0,
$$

where $A, B, C, D$ are analytic, has solutions if

$$
B^{2}-4 A C=2\left(A^{\prime} B-A B^{\prime}\right),
$$

a condition which is fulfilled for any $A$ and $B$ if $C$ is properly chosen, provided $A$ does not vanish.

Such linear equations, however, always have rather simple non-analytic solutions; if we express them in the form $F(z, \bar{z})$, as in (31), it results that $F$ is an analytic function of $z$ and a polynomial in $\bar{z}$, for linear equations of any order. However, these solutions are helpful also in obtaining ordinary analytic solu- 
tions, since it is shown that the analytic function $F(z, z-C)$ obtained by replacing $\bar{z}$ by $z-C$, is also a solution of the given differential equation.

Calugaréano also showed that any differential equation of the second order that has non-analytic solutions must be of the form

$$
w^{\prime \prime}+A(z, w) w^{\prime 2}+B(z, w) w^{\prime}+C(z, w)=0,
$$

where $A, B$, and $C$ are analytic functions of $z$ and $w$. It is true in this case also that if $F(z, \bar{z})$ is a solution of $(46)$, then $F(z, z-C)$ is an analytic solution of the same equation. These results are generalized to equations of higher order.

In his 1930 paper cited above, Calugaréano shows that the analytic integral of (46) is obtained from the non-analytic integral $F(z, \bar{z})$ by replacing $\bar{z}$ by $\gamma_{1} z+\gamma_{2}$.

15. Other Results. Conclusion. The results mentioned above have explicit reference to theorems on non-analytic functions. Two other directions exist in which associated results are to be found. In the first place, as is emphasized above, even the classical textbooks contain many theorems in which the hypothesis of analytic character is not made. A complete report on theorems satisfied by non-analytic functions would have to include all such theorems, which would not be appropriate here. Moreover, as the theory of non-analytic functions is becoming more widely known, more attention has been and will be paid to the question as to whether or not the hypothesis of analytic character is necessary, in any given theorem. I have called attention to several theorems in which such a hypothesis can be modified without essential change in the conclusion of the theorem. I have not tried to include all such instances in this paper. It is to be expected that a very large number of such cases will be found, as has been true in the similar case in the theory of functions of real variables.

Other notable omissions are caused through lack of space. In a list which follows, I have included the titles of some papers to which I have made no explicit references. In general, I have sought to include results which seemed to possess not only interest, but also a degree of apparent finality. Some results appear to be tentative in character, as is natural in a new field. 
The other direction of research associated with this paper, but not represented here, is the generalization of ideas inherent in the background of this paper, through extension to higher dimensional spaces, or to functions of other fundamental elements than points, or to hypercomplex numbers. A number of papers of this character have been published, and they are highly significant, but they seemed not to be an integral part of the discussion here, unless the field of discussion were to be extended unduly, in view of the limitation of space. That I myself have assisted in the publication of some of these is evidence enough that my present omission of them is not caused by any lack of personal interest.

It is my hope that the present paper may serve to acquaint many with some of the salient advances in the theory, to enable others to find a variety of results in one compact paper, and to give some indication of possible future research.

\section{BIBLIOGRAPHY}

G. Calugaréano, Sur les fonctions polygènes d'une variable complexe, Thesis, Paris, 1928.

G. Calugaréano, Comptes Rendus, vol. 186 (1928), p. 186; vol. 186 (1928), p. 930 ; vol. 186 (1928), p. 1406.

G. Calugaréano, Bulletin de la Société des Sciences de Cluj, vol. 14 (1928), pp. 357-361.

G. Calugaréano, Transactions of this Society, vol. 31 (1929), p. 372 ; vol. 32 (1930), p. 110.

J. E. Donahue, Concerning the geometry of the second derivative of a polygenic function, Columbia University Thesis, Hamburg, 1930.

E. R. Hedrick, L. Ingold, and W. D. A. Westfall, Theory of non-analytic functions of a complex variable, Journal de Mathématiques, (9), vol. 2 (1923), pp. 327-342.

E. R. Hedrick and L. Ingold, Analytic functions in three dimensions, Transactions of this Society, vol. 27 (1925), pp. 551-555.

E. R. Hedrick and L. Ingold, The Beltrami equations in three dimensions, Transactions of this Society, vol. 27 (1925), pp. 556-562.

E. R. Hedrick, On the derivatives of non-analytic functions, Proceedings of the National Academy, vol. 14 (1928), pp. 649-654.

E. R. Hedrick, On certain properties of non-analytic functions of a complex variable, Bulletin of the Calcutta Mathematical Society, vol. 20 (1928), pp. 109-124; see also this Bulletin, vol. 34 (1928), pp. 150, 435-6, 703; vol. 35 (1929), pp. 176, 599; vol. 36 (1930), pp. 59, 368, 801.

Lulu Hofmann and E. Kasner, Homographic circles or clocks, this Bulletin, vol. 34 (1928), pp. 495-503.

E. Kasner, $A$ new theory of polygenic (or non-monogenic) functions, Science, vol. 46 (1927), pp. 581-582. 
E. Kasner, General theory of polygenic (or non-monogenic) functions. The derivative congruence, Proceedings of the National Academy, vol. 13 (1928), pp. 75-82.

E. Kasner, Géométrie des fonctions polygènes, Atti del Congresso, Bologna, 1928, vol. 6, pp. 255-260.

E. Kasner, The second derivative of a polygenic function, Transactions of this Society, vol. 30 (1928), pp. 803-816.

E. Kasner, Note on the derivative circular congruence of a polygenic function, this Bulletin, vol. 24 (1928), pp. 561-565.

P. W. Ketchum, Analytic functions of hypercomplex variables, Transactions of this Society, vol. 30 (1928), pp. 641-667.

P. W. Ketchum and Ted Martin, Polygenic functions of hypercomplex variables, this Bulletin, vol. 38 (1932), pp. 66-72.

Edna E. Kramer, Polygenic functions of the dual variable $w=u+j v$, Columbia University Thesis, 1930; see also American Journal of Mathematics, vol. 52 (1930), pp. 370-376.

L. Lichtenstein, Sitzungsberichte, Berliner Mathematische Gesellschaft, 1910, pp. 84-100.

H. Looman, Ueber eine Erweiterung des Cauchy-Goursatschen Integralsatzes, Nieuw Achief voor Wiskunde, vol. 14 (1924), pp. 234-239.

P. Montel, Comptes Rendus, vol. 156 (1913), pp. 1820-1822; Annales de l'École Normale, (3), vol. 24 (1907), pp. 283-295.

M. Nicolesco, Fonctions complexes dans le plan et dans l'espace, Thesis, Paris, 1928; Comptes Rendus, vol. 185 (1927), p. 442.

D. Pompeiu, Sur la continuité des fonctions d'une variable complexe, Thesis, Paris, 1905.

D. Pompeiu, Sur une classe de fonctions d'une variable complexe, Rendiconti di Palermo, vol. 23 (1912), pp. 108-113; vol. 35 (1913), p. 277.

V. C. Poor, Residues of polygenic functions, Transactions of this Society, vol. 32 (1930), pp. 216-222.

H. Rademacher, Bemerkungen zu den Cauchy-Riemannschen Differentialgleichungen und zum Moreraschen Satz, Mathematische Zeitschrift, vol. 3 (1919).

G. E. Raynor, Generalization of the Beltrami equations to curved $n$-space, this Bulletin, vol. 33 (1927), pp. 435-439.

J. Ridder, Ueber den Cauchyschen Integralsatz für reelle und komplexe Funktionen, Mathematische Annalen, vol. 102 (1929), pp. 132-156.

J. Ridder, Ein Satz über iterierte Integrale und seine Anwendung zur Untersuchung der Analyticität komplexer Funktionen, Mathematische Zeitschrift, vol. 31 (1929), pp. 141-148.

J. Ridder, Ueber Analyticität komplexer Funktionen, Nieuw Archiv voor Wiskunde, (2), vol. 16 (1929), pp. 17-19.

J. Wolff, On the sufficient conditions for analyticity of functions of a complex variable, Report of the Toronto Congress, pp. 457-459; see also Nieuw Archiv voor Wiskunde, vol. 14 (1925),

The University of California at Los Angeles 